\title{
Some Observations on the Mechanics of
}

\section{Drops in Liquid-Liquid Systems}

\author{
E. R. ELZINGA, JR., and J. T. BANCHERO \\ University of Michigan, Ann Arbor, Michigan
}

\begin{abstract}
Data on the rate of rise and distortion of drops in several liquid-liquid systems have been collected. These are compared with correlations developed by other investigators, and in some cases discrepancies between observed and predicted values are noted which do not seem to be related to any easily measured physical property. However evidence is given showing that they are most likely due to the presence of surface-active materials. Photographs and shadowgraphs showing the nature of the flow around and behind moving drops are also included. The point of boundary-layer separation on the drop surface as indicated by these photographs has been related to the internal drop circulation. It is the shift in this point of separation which is the primary cause for the reduction in drag of liquid drops as compared with solid spheres. A previous theory for the breakup of rain drops explains the existing data for drop breakup in liquid-liquid systems.
\end{abstract}

A number of interesting observations on the mechanics of drops in liquid-liquid systems, which were made during an investigation of the rate of heat transfer to these drops (1), are presented in this paper.

The mechanics of the motion of drops and bubbles has been reviewed by Hughes and Gilliland (9) and more recently by Lane and Green (16). However since the time of these reviews a number of interesting papers have appeared. Drop formation has been studied by Keith and Hixon (12), as well as by Fujinawa, Maruyama and Nakaike (3). Drop velocities have been measured by $\mathrm{Hu}$ and Kintner (8), Klee and Treybal (13), Licht and Narasimhamurty (17), and Johnson and Braida (11). The motion of drops during acceleration has been studied by Pearcey and Hill (23), and Ingebo (10). Related material includes the papers by Linton and Sutherland (18), Sherwood and Wei (24), and Melhus and Terjesen (21) on the ef fect of surface-active materials on drop motion. In the present paper an attempt has been made not to cover subjects adequately discussed by the above authors.

\section{SUMMARY OF EXPERIMENTAL PROCEDURE}

Details of the equipment used in this investigation have been presented elsewhere (1). Briefly it consisted of a vertical test chamber 6 in. sq. and $2 \mathrm{ft}$. high. The front and back sides of this test section were made of glass, permitting visual and photographic observations. The continuous phase was maintained at a constant temperature by circulating it through an external heater and moved countercurrent to the drops at a rate which was small compared with the drop velocity.

E. R. Elzinga, Jr., is with the Esso Research and Engineering Company, Linden, New Jersey. Dame, Notre Dame, Indiana

Page 394
A single stream of drops was formed by forcing the dispersed phase through a water-jacketed nozzle. The tip at which the drops were formed was drilled and then bevelled at $45 \mathrm{deg}$., so that the edge was sharp and even. The drill size used for each nozzle is given in Table 1.

Photographs were made by illuminating the drops from behind with a high-speed $\left(10^{-\bar{s}}\right.$ sec. $)$ mercury arc. Shadowgraphs were made by placing a photographic plate on one side of the test section and discharging a capacitor through a short air gap on the opposite side. In most of the photographs and all of the shadowgraphs the motion of the fluid around the drops and in their wakes is clearly indicated. This is due to differences in the refractive index of the continuous phase in the region of the cold drops.

Drop velocity was obtained either from motion pictures or by calculation with the average distance between drops, as measured from photographs, and the drop frequency. The measured velocities were therefore relative to the test chamber and should be corrected for the continuouswas small enough to be negligible. In the range 0.5 to 5 drops/sec. no effect of drop-formation rate on velocity could be detected.

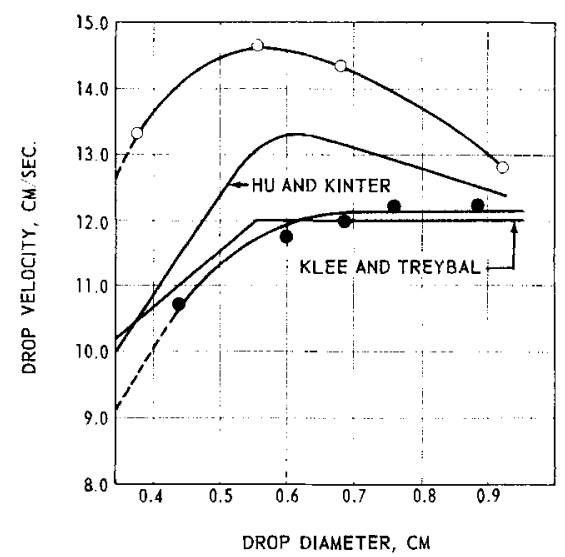

Fig. 1. Velocity of water drops in Dowtherm $A+E$, - with surface-active powder, o without surface-active powder. phase velocity. However the latter velocity
The average drop volume was calculated by dividing the dispersed phase flow rate by drop frequency. The diameter of a sphere of equivalent volume was used as the characteristic drop dimension.

The experimental data, pertinent physical properties, and calculated values of the Reynolds number and drag coefficient for the drops are given in Table 2.

\section{DROP VELOCITIES}

After the drops form and break away from the tip, there is a short period in which they accelerate to their terminal velocity. From motion pictures it was found that the drops always had reached at least $95 \%$ of terminal velocity after traveling 2 in. The drop velocity after this initial period of acceleration was constant enough so that little variation could be detected during the remainder of the drop rise. The variation of drop velocity with diameter was typical of liquid-liquid systems. This is illustrated in Figure 1, which shows the increase in velocity with diameter for water drops in an equal volume mixture of Dowtherm A and Dowtherm E. The upper curve shows the characteristic maximum associated with the start of drop oscillation. The lower curve in this figure is for drops in the same system but with a surface-active powder present. In this case the point at which oscillation occurs is not obvious as there does not seem to be a noticeable maximum. The difference between these two sets of data will be discussed in more detail in the next section.

A comparison of the measured velocities with two existing correlations is also shown in Figure 1. The Klee and Treybal correlation (13) gives a very close fit to the lower set of data. On the other hand the $\mathrm{Hu}$ and Kintner correlation (8) gives results intermediate between the two sets of data.

It is interesting that neither of these correlations predicts velocities as high

$\begin{array}{cc}\text { Table 1. List of Nozzles Used } \\ \text { Letter } & \text { Drill } \\ \text { designation } & \text { diameter (in.) } \\ \text { a } & 0.022 \\ \text { b } & 0.0313 \\ \text { c } & 0.0625 \\ \text { d } & 0.0938 \\ \text { e } & 0.125 \\ \text { f } & 0.250\end{array}$


Table 2. Summary of Experimental Data

System-water drops in Dowtherm E

$$
\begin{array}{ll}
\sigma \text { at } 25^{\circ} \mathrm{C} .=39.5 \text { dynes } / \mathrm{cm} . & \sigma \text { at } 80^{\circ} \mathrm{C} .=35.8 \\
\rho \text { at } 60^{\circ} \mathrm{C} .=1.268, \mathrm{~g} . / \mathrm{cc} . & \rho \text { at } 80^{\circ} \mathrm{C} .=1.253 \\
\mu \text { at } 60^{\circ} \mathrm{C} .=0.00843 \text { poises } & \mu \text { at } 80^{\circ} \mathrm{C} .=0.00758
\end{array}
$$

\begin{tabular}{|c|c|c|c|c|c|c|c|c|}
\hline $\begin{array}{l}\text { Run } \\
\text { no. }\end{array}$ & $\begin{array}{c}\text { Continuous } \\
\text { phase } \\
\text { temp., } \\
{ }^{\circ} \mathrm{C} .\end{array}$ & $\begin{array}{l}\text { Av. } \\
\text { drop } \\
\text { temp. } \\
{ }^{\circ} \mathrm{C} .\end{array}$ & $\begin{array}{c}\text { Drop } \\
\text { diam., } \\
\text { cm. }\end{array}$ & $\begin{array}{c}\text { Drop } \\
\text { vel., } \\
\mathrm{cm} . / \mathrm{sec} .\end{array}$ & $\begin{array}{c}\text { Drop } \\
\text { freq }^{3} \text { cy } \\
\text { (sec. })^{-1}\end{array}$ & $\begin{array}{c}\text { Ratio } \\
\text { of } \\
\text { diams. }\end{array}$ & $\begin{array}{l}\text { Drop } \\
\text { Reynolds } \\
\text { number }\end{array}$ & $\begin{array}{l}\text { Drop } \\
\text { drag } \\
\text { coef. }\end{array}$ \\
\hline $\mathrm{A} 19 \mathrm{e}$ & 60.3 & 45.0 & 0.616 & 13.3 & 0.996 & 1.19 & 1,229 & 1.01 \\
\hline A20d & 59.2 & 37.1 & 0.590 & 13.6 & 1.12 & 1.30 & 1,192 & 0.9 \\
\hline $\mathrm{A} 21 \mathrm{c}$ & 59.0 & 41.0 & 0.504 & 13.6 & 1.38 & 1.19 & 1,019 & 0.78 \\
\hline $\mathrm{A} 22 \mathrm{~b}$ & 59.1 & 49.0 & 0.420 & 12.5 & 0.984 & 1.15 & 781 & 0.78 \\
\hline
\end{tabular}

System-water drops in Dowtherm $\mathrm{A}+\mathrm{E}$ $\begin{array}{ll}\sigma \text { at } 25^{\circ} \mathrm{C} .=38.5 \text { dynes } / \mathrm{cm} . & \sigma \text { at } 80^{\circ} \mathrm{C}=35.5 \\ \rho \text { at } 60^{\circ} \mathrm{C} .=1.158, \mathrm{~g} . / \mathrm{cc} . & \rho \text { at } 70^{\circ} \mathrm{C} .=1.148 \\ \mu \text { at } 60^{\circ} \mathrm{C} .=0.0111 \text { poises } & \mu \text { at } 70^{\circ} \mathrm{C}=0.00987\end{array}$

$\begin{array}{lrlllllrl}\text { B4b } & 58.1 & 43.2 & 0.442 & 10.7 & 1.95 & 1.12 & 482 & 0.73 \\ \text { B5c } & 58.0 & 35.0 & 0.600 & 11.7 & 1.44 & 1.22 & 713 & 0.81 \\ \text { B6d } & 58.0 & 33.1 & 0.695 & 11.9 & 1.41 & 1.25 & 847 & 0.89 \\ \text { B7e } & 58.7 & 32.2 & 0.765 & 12.1 & 1.57 & 1.39 & 951 & 0.96 \\ \text { B8f* * } & 58.2 & 33.0 & 0.890 & 12.1 & 1.01 & 1.67 & 1,097 & 1.11 \\ \text { B13c* * } & 59.0 & 45.1 & 0.560 & 14.6 & 1.83 & 2.24 & 844 & 0.49 \\ \text { B15d * } & 59.1 & 43.8 & 0.682 & 14.3 & 1.84 & 2.08 & 1,009 & 0.63 \\ \text { B16a } & 58.8 & 47.9 & 0.380 & 13.3 & 1.97 & 1.60 & 521 & 0.41 \\ \text { B17f* * } & 58.9 & 38.0 & 0.927 & 12.8 & 0.906 & & 1,224 & 1.05\end{array}$

System-ethylene glycol in Dowtherm A $+\mathrm{E}$ $\sigma$ at $25^{\circ} \mathrm{C} .=9.0$ dynes $/ \mathrm{cm} . \quad \sigma$ at $80^{\circ} \mathrm{C} .=9.0$

$\begin{array}{lllllllll}\text { C1f } & 59.0 & 42.0 & 0.881 & 6.95 & 0.449 & 2.08 & 632 & 1.20 \\ \text { C2d } & 59.3 & 45.9 & 0.673 & 7.75 & 0.622 & 1.52 & 539 & 0.78 \\ \text { C3a } & 59.0 & 53.9 & 0.464 & 7.00 & 0.600 & 1.21 & 335 & \mathbf{0 . 7 2}\end{array}$

System-ethylene glycol in Dowtherm E $\sigma$ at $25^{\circ} \mathrm{C} .=8.5 \mathrm{dynes} / \mathrm{cm} . \quad \sigma$ at $80^{\circ} \mathrm{C} .=7.96$

$\begin{array}{llllccccc}\text { D1b } & 58.1 & 56.0 & 0.288 & 9.31 & 1.60 & 1.31 & 396 & 0.62 \\ \text { D2d } & 59.0 & 51.3 & 0.437 & 9.28 & 1.32 & 1.49 & 604 & 0.93 \\ \text { D3c } & 58.8 & 53.8 & 0.369 & 10.4 & 1.78 & 1.57 & 570 & 0.64\end{array}$

System-water drops in finol

$$
\begin{array}{ll}
\sigma \text { at } 25^{\circ} \mathrm{C} .=50.5 \mathrm{dynes} / \mathrm{cm} . & \sigma \text { at } 80^{\circ} \mathrm{C} .=44.6 \\
\rho \text { at } 60^{\circ} \mathrm{C} .=0.823 \mathrm{~g} . / \mathrm{cc} . & \mu \text { at } 60^{\circ} \mathrm{C} .=0.0710 \text { poises }
\end{array}
$$

$\begin{array}{lllllllll}\text { E1c } & 60.1 & 42.0 & 0.645 & 16.2 & 0.583 & 1.23 & 121 & 0.66 \\ \text { E2c } & 60.0 & 44.1 & 0.655 & 16.8 & 0.769 & 1.27 & 128 & 0.61 \\ \text { E3a } & 61.0 & 51.8 & 0.450 & 9.65 & 1.08 & 1.06 & 51.0 & 1.27 \\ \text { E4b } & 59.4 & 47.8 & 0.523 & 13.8 & 0.729 & 1.14 & 82.8 & 0.72 \\ \text { E5d } & 60.0 & 45.5 & 0.729 & 18.1 & 0.691 & 1.57 & 153 & 0.59 \\ \text { E6e } & 59.1 & 38.3 & 0.804 & 18.2 & 0.712 & 1.61 & 167 & 0.65 \\ \text { E7f * } & 61.0 & 38.0 & 0.964 & 18.3 & 0.579 & 1.86 & 207 & 0.78\end{array}$

System-ethylene glycol in finol $\sigma$ at $25^{\circ} \mathrm{C} .=19.5$ dynes $/ \mathrm{cm} . \quad \sigma$ at $80^{\circ} \mathrm{C} .=18.4$

$\begin{array}{llllccccc}\text { F1b } & 59.2 & 53.8 & 0.330 & 9.03 & 1.77 & 1.11 & 34.1 & 1.70 \\ \text { F2c } & 59.0 & 52.1 & 0.376 & 11.1 & 1.19 & 1.24 & 47.7 & 1.29 \\ \text { F3d } & 60.4 & 50.0 & 0.423 & 12.5 & 1.11 & 1.33 & 61.3 & 1.16 \\ \text { F4e } & 60.3 & 47.3 & 0.473 & 13.9 & 1.17 & 1.59 & 76.3 & 1.05\end{array}$

* Additional data on some of these systems are reported in reference 1.

* A double asterisk following the run number indicates that the drops were observed to oscillate rapidly between prolate and oblate spheroids.

Note: Letter suffixes refer to nozzle used, see Table 1. as those obtained with uncontaminated water drops. This suggests the possibility that the systems upon which the correlations were based contained traces of surface-active materials. Furthermore these contaminants need not have any measurable effect on the physical properties of the system. In the present case for example no change in interfacial tension, bulk and surface viscosities, or density could be discerned when the surface-active powder was added to the system.

Surface viscosity exists at a liquidliquid interface if the interface itself contributes to the resistance of a body moving in the plane of the interface. Since surface-active materials concentrate at the interface, a change in surface viscosity might be expected when one of these agents is present. However in the present work no change could be detected after addition of the surface-active powder. The technique used to measure this quantity was similar to that described by Brown (2) who utilized a rotational type of torsional viscometer with a ring bob. It is possible that this technique is not applicable to films composed of solid particles.

The above observations on the effect of surface-active materials have been previously made by Garner and coworkers $(4,5)$. These authors observed visually the effect of surfaceactive materials on internal drop circulation. They found that as concentration was increased, circulation was damped out starting from the rear pole of the drop. Here, as in the present work, these effects took place at concentrations of surface-active material low enough so that there was little or no measurable change in interfacial tension.

The surface-active powder, in this work, was believed to be alumina picked up from the inside of aluminum tubing. This contaminant was believed to be present in all runs prior to and including B8f. A check on the properties of alumina showed that it is hydrophilic and would therefore collect on the inside surface of water drops. For this reason the powder would not tend to wash off the drops but would instead build up on the surface, starting at the rear stagnation point. This film on the drop surface is capable of supporting shear. Thus internal circulation is inhibited, and the net result is an increase in the drop drag coefficient.

Unfortunately a positive identification of the surface-active powder was not obtained. However with respect to drop mechanics it behaved exactly like alumina. This was demonstrated 


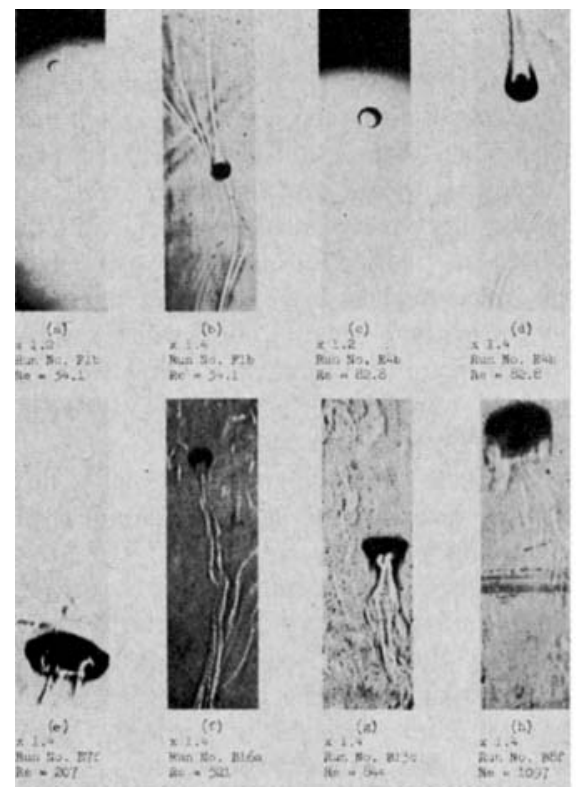

Fig. 2. Photographs and shadowgraphs of moving drops.

by injecting a dilute slurry of finely ground alumina ahead of the drop nozzle. When this slurry reached the nozzle, the drops reverted to their original lower velocity.

Figures 2 and 3 are a series of photographs and shadowgraphs showing the appearance of the moving drops and their wakes. Photographs $a$ through $d$ of Figure 3 are of contaminated water drops and correspond to the lower set of data in Figure 1; photographs $e$ through $h$ correspond to the higher set of data in Figure 1.

All of the experimentally determined drag coefficients are plotted against the corresponding Reynolds numbers in Figure 4. This figure also includes experimentally determined curves for the drag on spheres and disks. The figure shows that the trend of experimental data for small drops is essentially the same as that for solid spheres. However the absolute value is much lower in some cases than that for solid spheres. As the drop size increases, drop deformation increases rapidly, and the curves pass through a minimum. At still larger sizes, drop oscillation occurs, and the drag coefficient increases rapidly. This rapid increase in drag coefficient proceeds until the drop finally ruptures. The latter point was not reached in any of the systems studied in the present work.

Stoke's law for solid spheres and a similar relation for nonviscous liquid spheres (14) have been extrapolated and plotted as broken and dashed lines respectively in Figure 4. A comparison of the broken line with the experimentally determined curve for solid spheres gives an approximation to that portion of total drag due to viscous drag. The difference between the

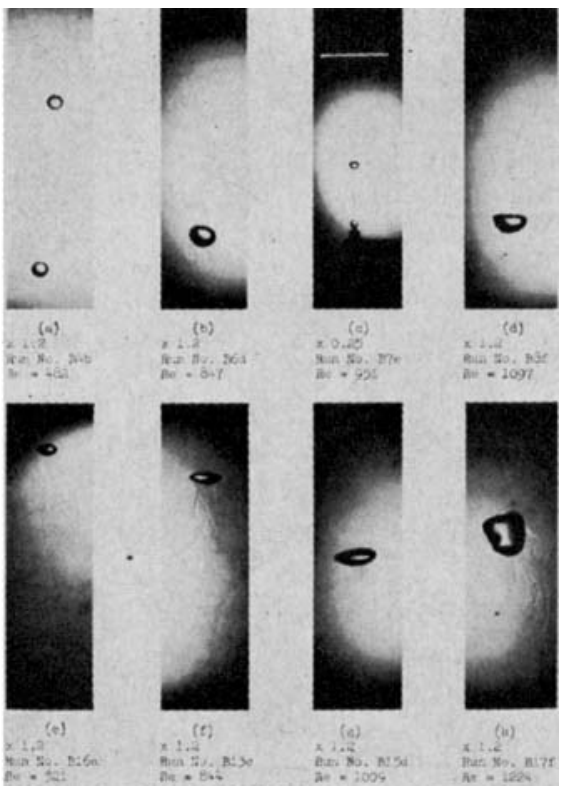

Fig. 3. Photographs and shadowgraphs of moving drops.

broken line and the dashed line compared with the experimental curve for solid spheres indicates the fraction of the total drag due to skin friction. It can be seen that only a small portion of the total drag is due to the skin friction in the range of Reynolds numbers considered here.

Almost all of the previous investigations of this type have noted cases in which the observed drag coefficients were lower than those for solid spheres. This difference was usually attributed entirely to the reduction in skin friction caused by internal circulation. However in this range of Reynolds numbers the complete loss of skin friction would account for at most only a $10 \%$ reduction in the drag coefficient. This percentage reduction can be estimated by taking the difference between the two curves for viscous How and dividing this by the experimentally determined drag coefficient for solid spheres. The observed reduction in drag coefficient is as high as $40 \%$ (water drops in Finol). Therefore internal circulation must have an additional effect on drag. The probable nature of this additional effect is discussed in the next section.

\section{INTERNAL-DROP CIRCULATION}

Mention has been made of the connection between the degree of internal circulation and the lowering of drop drag coefficient below that for solid spheres. It was also shown that only a small portion of this could be explained by the reduction in skin friction. Therefore internal circulation must also reduce the net effect of form drag and/or induced drag. The manner in which this reduction takes place

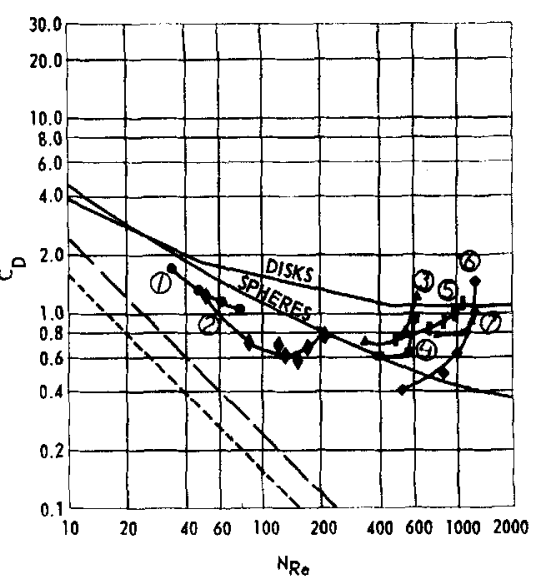

Fig. 4. Drag coefficients for systems studied, 1) ethylene glycol in finol, 2) water in finol, 3) ethylene glycol in Dowtherm A + E, 4) ethylene glycol in Dowtherm E, 5) contaminated water in Dowtherm $A+E, 6)$ uncontaminated water in Dowtherm A $+E, 7)$ water drops in Dowtherm $E,-$ Stokes law for solid spheres, -... - viscous flow for liquid spheres $\left(\mu_{\mathrm{d}}=\right.$ $0)$, experimental.

was indicated by photographs of the drops and their wakes.

Reference is again made to Figure 3 , containing the photographs of contaminated and uncontaminated water drops in Dowtherm A $+\mathbf{E}$. It will be recalled that the velocity of the uncontaminated drops was considerably higher than that for the contaminated drops. This suggests that the internal circulation is higher in the uncontaminated case. This is further substantiated by heat transfer measurements made in this system which showed that the internal heat transfer coefficients were considerably higher for the uncontaminated drops.

It will be noted in most of the photographs of Figure 3 that the point of boundary-layer separation and the flow pattern in the wake is visible. The important difference between the two sets of data is the shift in the point of separation from the front half of the drop to the back half. This can be most easily seen by comparing photographs $b$ and $f$, which, are at approximately the same Reynolds number. The shift is believed to have been caused by the higher degree of internal circulation in the uncontaminated drops. This causes the liquid in the boundary layer to have a higher average velocity and thus be better able to overcome the adverse pressure gradient which occurs in the region of the drop equator. For solid spheres in this range of Reynolds numbers separation occurs at about $9 \mathrm{deg}$. forward from the equator.

The result of this shift in point of boundary-layer separation is to reduce the size of the wake behind the drop. This in turn produces a corresponding 
TABLe 3. EFfect of INTERNaI Crrculation on the Point of Separation

$\begin{array}{lc}v . / u & \Delta \theta \text { (deg.) } \\ 0 & 0 \\ 0.1 & 1.2 \\ 0.2 & 2.1 \\ 0.3 & 2.7 \\ 0.5 & 3.8 \\ 0.7 & 4.8 \\ 0.9 & 5.7 \\ 1.5 & 7.7\end{array}$

reduction in induced drag. Induced drag is that portion of the total drag caused by the transfer of energy to the vortex system in the drop wake.

The effect of degree of internal circulation on the drag coefficient is illustrated by comparing curves 5 and 6 in Figure 4. These curves are, again, for contaminated and uncontaminated water drops in Dowtherm A and E and correspond to the velocity data of Figure 1 and the photographs of Figure 3 . It will be noted that the drag coefficient for contaminated drops, curve 5 , is nearly twice that for uncontaminated drops. It will also be noted that a portion of the curve for uncontaminated drops falls below that for solid spheres even though these drops are greatly distorted.

Internal circulation can also explain the low drag coefficients (compared with solid spheres) for ethylene glycol drops and water drops in Finol [curves (1) and (2) of Figure 4]. As has been mentioned previously skin friction can at most cause only a $10 \%$ reduction in drag coefficient. However shadowgraph $e$ of Figure 2 for water drops in Finol clearly illustrates that separation takes place well to the rear of these drops. Thus the remainder of the reduction is due to a reduction in induced drag.

\section{THEORETICAL ANALYSIS OF SHIFT IN POINT OF BOUNDARY-LAYER SEPARATION}

The magnitude of the shift in the point of separation can be estimated by solving the appropriate boundarylayer equation. Meksyn (20) has derived this equation for laminar flow around solid spheres. For the front portion of the sphere his equation reduces to the following expression for the stream function:

$f^{\prime \prime \prime}+f^{\prime \prime}-\frac{\cos \theta}{\sin ^{\theta}-\frac{\theta}{2}}\left(1-f^{\prime 2}\right)=0$

The corresponding boundary conditions are

$$
\begin{array}{lll}
f=\frac{\partial f}{\partial \eta}=0 & \text { at } & \eta=0 \\
\frac{\partial f}{\partial \eta}=1 & \text { at } & \eta=\infty
\end{array}
$$

Here $\eta$ and $f$ are defined by

$\eta=\frac{1}{2}\left(\frac{2 u}{\nu \gamma}\right)^{1 / 2} \beta$, and

$$
\Psi=(2 \nu u \gamma)^{1 / 2} f
$$

In equation form $\beta$ and $\gamma$ are

$$
\beta=\frac{1}{2}\left(\frac{r^{2}}{a}-\frac{a^{2}}{r}\right) \sin ^{2} \theta
$$

and

$$
\begin{array}{r}
\gamma=\frac{3 a}{2}+\alpha=\frac{3 a}{2}+ \\
\left(r+\frac{a^{3}}{2 r^{2}}\right) \cos \theta
\end{array}
$$

In other words $\beta$ is the inviscid stream function for flow around a sphere when the undisturbed velocity is 1 , and $\gamma$ is a constant plus the corresponding velocity potential.

The polar angle is measured from the rear stagnation point.

For circulating drops the continuous-phase velocity is not zero at the drop surface. Therefore the boundary conditions at $\eta=0$ become

$$
f=0 \text { and } \frac{\partial f}{\partial \eta}=v_{s} / u
$$

Equation (1) can now be solved at any point $\theta$ on the drop surface. The value of $\theta$ for which $f^{\prime \prime}(0)=0$ corresponds to the point of separation. Thus the effect of $v_{s}$ on this point can be determined.

Solutions of Equation (1) were obtained with an electronic differential analyzer. In this computer the dependent variable $f$ was represented by a voltage, while the independent variable $\eta$ was set proportional to time. For a particular value of $v$, the setting on the potentiometer representing cos $\theta / \sin ^{2}(\theta / 2)$ was varied, and a plot of $f^{\prime}$ vs. time was obtained. The correct setting on this potentiometer was taken as the one which produced the most rapid convergence of $f^{\prime}$ to unity.

The calculated value of $\theta$ at the point of separation for zero surface velocity (solid sphere) is $83 \mathrm{deg}$. For a finite surface velocity the predicted point of separation changes by the amount shown in Table 3. Here the change in the angle $\theta$ at which separa. tion occurs is given as a function of the ratio of surface velocity to freestream velocity. When the surface velocity is equal to the free-stream velocity, the separation point should move back about 6 deg.

The predicted shift in separation point appears to be somewhat less than that actually observed. However this difference could be due to assumptions made in deriving the boundary-layer equation and to the fact that the drops are in most cases far from being spherical. In any case the order of magnitude of the calculated shift is such that it gives credence to the theory that it is caused primarily by internal circulation.

\section{DROP DISTORTION}

A moving drop is distorted by the local forces acting over its surface. These forces are caused by differences in hydrostatic head, external and internal fluid motion, interfacial tension, and in some cases the drag of external liquid over the surface of the drop. Direct distortion by the latter phenomenon can occur only if the drop surface is composed of an adsorbed film capable of supporting shear. For nonoscillating drops the sum of these forces at any point on the surface is zero.

Neglecting the effect of internal circulation Hughes and Gilliland (9) have derived an equation for predicting the ratio of minor to major drop diameters. Making this same assumption McDonald (19) was able to calculate pressure distributions around rain drops from their shapes in free fall through air. In this latter work the effect of internal circulation was shown to be negligible.

The assumption that internal circulation has little effect on distortion probably holds for most systems where drops are dispersed in a gas. However the application of the Hughes and Gilliland equation to liquid-liquid systems predicts values much lower than those actually observed.

Distortion data from the present work are shown in Figure 5, where the ratio of major to minor drop diameter is plotted against the average diameter. These data for water drops in Dowtherm $\mathrm{A}+\mathrm{E}$, obtained during

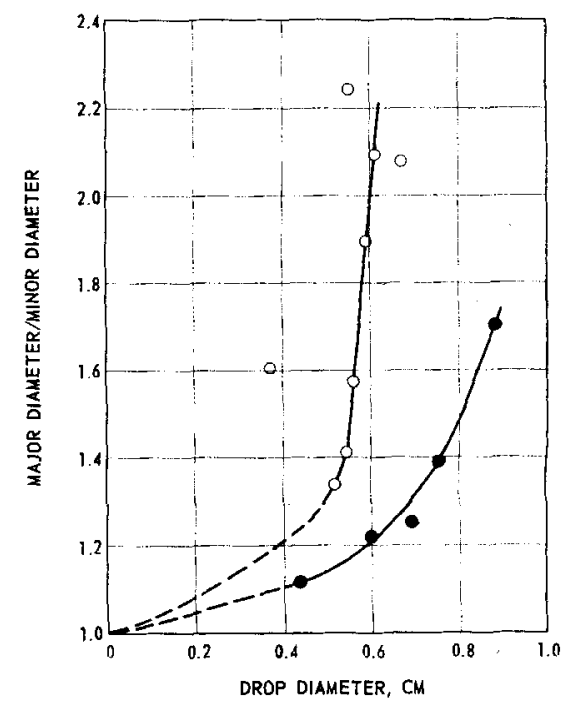

Fig. 5. Distortion of water drops in Dowtherm $A+E$, o without surface-active powder, with surface-active powder. 
the two series of runs previously mentioned, correspond to the velocity data of Figure 1 and the photographs shown in Figure 3. The large increase in distortion due to more rapid internal circulation in the series where no surface active powder was present is easily seen. This is particularly apparent at drop diameters above 0.5 $\mathrm{cm}$., where even though the drop velocities for the two series are approaching each other, the difference between their distortions is increasing. This marked difference in distortion again emphasizes the importance of factors whose presence may not be detected through easily measured properties such as static interfacial tension, viscosity, and density.

\section{MECHANISM OF DROP BREAKUP}

A great deal of experimental and theoretical work has been done on the breakup of moving drops. Various mechanisms by which this can occur have been summarized by Hinze (7). This summary covers a number of important cases which are common to liquid-gas as well as liquid-liquid systems. However it omits one important phenomenon of drop breakup which has been observed in both systems, the breakup caused by oscillations of large amplitude. It has been observed by $\mathrm{Hu}$ and Kintner (8) for liquid-liquid systems and by Gunn (6) for raindrops falling through air. Gunn has suggested a mechanism for this breakup of raindrops, and as will be seen below it also applies to the breakup of drops in liquid-liquid systems.

It is well known that there is a periodic discharge of material from the vortex ring formed in the wake of blunt bodies. This discharge momentarily reduces the size of the wake and hence the drag coefficient. For drops the resulting acceleration causes the drop to oscillate. Gunn has suggested that the amplitude of these oscillations becomes large enough to rupture the drop when their frequency matches the natural frequency of the drop.

The natural frequency of oscillation for a drop can be estimated with a relation (15) developed for liquid spheres. The primary mode of this oscillation is given by the following equation:

$$
F=\sqrt{\frac{192 \sigma}{\left(3 \rho_{d}+2 \rho\right) D^{3}}}
$$

As can be seen the natural frequency of a liquid drop decreases as its size increases. The discharge frequency of material in the wake of steel spheres dropping through water has been measured by Möller (22) and was found to increase with increasing di-

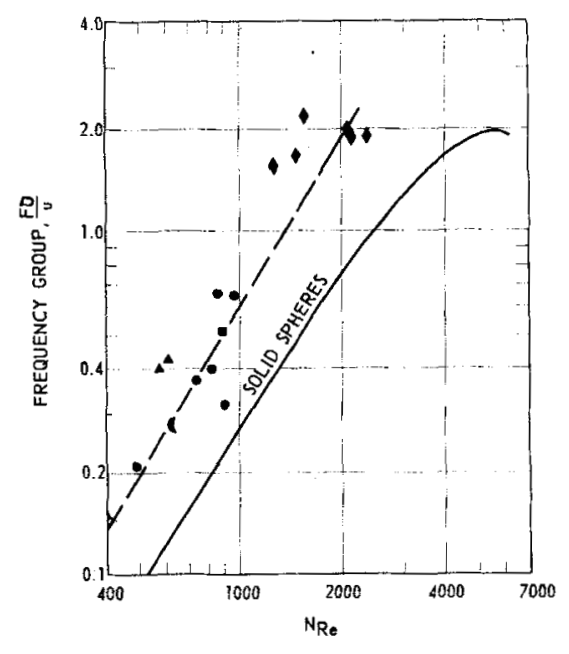

Fig. 6. Vortex diseharge frequency for drops, - - Equation (3), _- Reference 22, Solid spheres, Diamond: Reference 8, Solid Square: water drops in Dowtherm $E$, Solid Circle: water drops in Dowtherm A $+E$, Solid Half Circle: ethylene glycol in Dowtherm A + , Solid Triangle: ethylene glycol in Dowtherm E.

ameter. It is therefore possible that for a particular system a drop size may exist such that the natural frequency of the drop matches the frequency of vortex discharge. A line through Moller's data, plotted as a dimensionless frequency group vs. the Reynolds number, is shown in Figure 6.

In the present work the frequency of discharge into the wake of the drops could be estimated. This was done by dividing the drop velocity by the distance between the enlargements in the wake as measured from the shadowgraphs. Shadowgraph $g$ of Figure 2 is an example of this type of wake. The resulting dimensionless frequency group is plotted against the corresponding Reynolds number in Figure 6. It can be seen that the data follow the same trend as those for solid spheres. The displacement of these data from the line for solid spheres is most likely caused by drop distortion. This is particularly true at the higher Reynolds numbers.

The drop-breakup data of $\mathrm{Hu}$ and Kintner (8) can be used to test the theory mentioned previously. These data are for six organic liquids in water and represent the drop size and velocity at which breakup occurs. When one uses Equation (2), the natural frequency of these drops can be estimated and the dimensionless frequency group computed. These calculated values are plotted in Figure 6 . It can be seen that they are consistent with the present work and that of Möller. This agreement indicates that the vortex discharge frequency matched the natural frequency of these drops at the time of breakup.
To estimate the critical drop diameter (at which breakup occurs) the following procedure can be used. The dashed line in Figure 6 has been fitted, by the method of least squares, to the experimental data and the equation found to be

$$
\frac{F D}{u}=3.12 \times 10^{-6} N_{R \varepsilon}^{1.8}
$$

This equation can be used in conjunction with Equation (2) and a suitable relation for the drop velocity to solve for the critical drop diameter. Since it is believed that the dimensionless frequency group for drops levels off in much the same way as it does for solid spheres, Equation (3) should not be used above a Reynolds number of 2,000 . Instead a constant value of about 1.9 should be used for the dimensionless frequency group. Also because viscosity affects the natural frequency of oscillation and is not accounted for in Equation (2) this relation should not be applied to extremely viscous drops.

\section{CONCLUSIONS}

The results of the present investigation show that drag coefficients for drops cannot be related entirely to easily measured physical properties. This is because the presence of small quantities of surface-active materials and possibly the method of formation are very important. It has been shown that internal circulation causes a shift in the point of boundary-layer separation on the drop surface. This in turn streamlines the drop and causes a substantial decrease in the drop drag coefficient below that for solid spheres.

Surface-active materials slow down internal circulation causing the point of separation to remain near the equator of the drop. In this case the drag coefficient is higher, and if the drop is also nonoscillating, it approaches the value for solid spheres. Internal circulation also causes a marked increase in drop distortion. Data on the breakup of free-falling drops have been related to the natural frequency of drop oscillation and the frequency of vortex shedding in the drop wake.

\section{NOTATION}

$a=$ drop radius, $\mathrm{cm}$.

$C_{D}=\operatorname{drag}$ coefficient, $4 D\left|\rho_{d}-\rho\right| g /$ $3 \rho u^{2}$

$D=$ drop diameter, $\mathrm{cm}$.

$F=$ frequency, $(\text { sec. })^{-1}$

$f \quad=$ stream function for boundary layer flow around the front half of a sphere

$r \quad=$ radial distance, $\mathrm{cm}$.

$N_{R e}=$ drop Reynolds number, $D u / \nu$ 
$u=$ drop velocity, cm./sec.

$v_{3}=$ local velocity at surface of drop, cm./sec.

\section{Greek Letters}

$\alpha \quad=$ velocity potential for irrotational flow around sphere, $\mathrm{cm}^{4} / \mathrm{sec}$.

$\beta=$ stream function for irrotational flow around sphere cc./ sec.

$\gamma=$ defined in discussion after Equation (1)

$\eta=$ defined in discussion after Equation (1)

$\theta \quad=$ polar angle measured from rear stagnation point

$=$ viscosity, poises

$=$ kinematic viscosity, stokes

$=$ continuous phase density, g./ cc.

$\rho_{\text {it }}=$ dispersed phase density, g./ cc.

$\sigma \quad=$ interfacial tension, dynes $/ \mathrm{cm}$. or g./sec. ${ }^{2}$

$\Psi \quad=$ stream function for boundary layer flow around sphere written in terms of the coordinates $\alpha$ and $\beta$

\section{LITERATURE CITED}

1. Elzinga, E. R., and T. T. Banchero, Chem. Eng. Progr. Symposium Ser. No. 29, 55, 149-61 (1959).

2. Brown, A. G., W. C. Thuman, and J. W. McBain, J. Colloid Sci., 8, 491507 (1953).

3. Fujinawa, K., T. Maruyama, and $\mathrm{Y}$. Nakaike, Chem. Eng. (Japan), 21, 194-200 (1957).

4. Garner, F. H., and A. R. Hale, Chem. Eng. Sci., 2, 157-63 (1953).

5. Garner, F. H., and A. H. P. Skelland, ibid., 4, 149-58 (1955).

6. Gunn, R., J. Geophysic. Research, 54, 383-5 (1949).

7. Hinze, J. O., A.I.Ch.E. Journal, 1, 289-95 (1955).

8. Hu, S., and R. C. Kintner, ibid., pp. 42-8 (1955).

9. Hughes, R. R., and E. R. Gilliland, Chem. Eng. Progr., 48, 497-504 (1952).

10. Ingebo, R. D., Natl. Advisory Comm. Aeronaut. Tech. Note 3762 (1956).

11. Johnson, A. I., and L. Braida, Can. J. Chem. Eng., 35, 165-72 (1957).

12. Keith, F. W., Jr., and A. N. Hixon, Ind. Eng. Chem., 47, 258-67 (1955).
13. Klee, A. J., and R. E. Treybal, A.I.Ch.E. Journal, 2, 444-7 (1956).

14. Lamb, H., "Hydrodynamics," 6 ed, pp. 600-2, Dover Publications, New York (1945).

15. Ibid., pp. $450-51$.

16. Lane, W. R., and H, L. Green, "Surveys in Mechanics," pp. 162-215, Cambridge Univ. Press, New York (1956).

17. Licht, William, and G. S. R. Narasimhamurty, A.I.Ch.E. Journal, 1, 366-73 (1955).

18. Linton, M., and K. L. Sutherland, Proc. Internatl. Congr. Surface Activity, 2nd, London, 1, 149-57 (1957).

19. McDonald, J. E., J. Meteorology, 11, 478-94 (1954).

20. Meksyn, D., Proc. Roy. Soc., 194A, 218-28 (1948).

21. Melhus, B. J., and S. G. Terjesen, Chem. Eng. Sci., 7, 83-88 (1957).

22. Möller, W., Physik. Z., 39, 64 (1938).

23. Pearcey, T., and G. W. Hill, Australian J. Phys., 9, 19-30 (1956).

24. Sherwood, T. K., and J. C. Wei, Ind. Eng. Chem., 49, 1030-4 (1957).

Manuscript received February 12, 1959; revision received February 9, 1961; paper accepted February 9, 1961 .

\section{Application of the Kihara Potential to}

\section{High Pressure Phase Equilibria}

\section{J. M. PRAUSNITZ and R. N. KeELeR}

University of California, Berkeley, California

This paper is concerned with the virial equation of state as applied to vapor mixtures at moderate densities. The required virial coefficients for small molecules can usually be estimated from generalized corresponding states correlations, but these correlations are not sufficient for mixtures which contain one very heavy and one very light component. It is shown that for such mixtures the necessary virial coefficients can be calculated by statistical thermodynamics with a potential function proposed by Kihara. This type of calculation is especially useful at low temperatures where virial coefficients have not been measured because of large experimental difficulties. Application of the Kihara potential is demonstrated with the computation of $K$ values for heavy components for several mixtures at advanced pressures; good agreement with experimental results is obtained.

The virial equation may in some cases be used to test data for thermodynamic consistency, and for illustration it is shown that recently reported $K$ data for propane in compressed hydrogen are thermodynamically inconsistent and too large by about two orders of magnitude.

Experimental studies in high-pressure fluid-phase equilibria are numerous and have appeared in the technical literature for many years. However very little effort appears to have been expended on the application of theoretical concepts to the problem of testing, correlating, and eventually predicting high-pressure phase-equilibrium relationships. This is unfortunate, primarily for two reasons. First, it is inconceivable that complete experimental data will ever become available for the almost infinite number of binary and multicomponent systems which are composed of the various gases and liquids of technical interest today, not to mention the even larger number of such fluids which may be of interest in the future. As a result it becomes necessary to carefully obtain data for a few representative systems and then to interpret these data with as much physical insight as possible. Such interpretation, to be most useful, must be guided by theoretical concepts not only because such concepts help to understand what intermolecular forces are responsible for the observed phase be- havior but also because an interpretation or correlation based only on empirical grounds cannot be successful when data are available for only a very limited number of systems.

Second, the application of theoretical methods enables the experimentalist to test his results for internal and thermodynamic consistency. At present such testing is almost never done by experimentalists in the high-pressure field, and consequently inaccurate data have been reported and have been uncritically accepted by the scientific and engineering community. As a result empirical correlations based on faulty data extend the error by producing incorrect predictions for systems for which no data are as yet available. Occasionally a sound correlation scheme may fail correctly to predict the apparent behavior of a given system, and the scheme is therefore rejected, due not to any fault in the correlation method but rather to the gross inaccuracy of the experimental data. An example of such a case is given towards the end of this paper. 